\title{
Breakdown of the mean-field approximation in a wealth distribution model
}

\author{
M Medo \\ Physics Department, University of Fribourg, Pérolles, 1700 Fribourg, \\ Switzerland \\ and \\ Department of Mathematics, Physics and Informatics, Mlynská dolina, \\ 84248 Bratislava, Slovak Republic \\ E-mail: matus.medo@unifr.ch
}

\begin{abstract}
One of the key socioeconomic phenomena to explain is the distribution of wealth. Bouchaud and Mézard (2000 Physica A 282 536) have proposed an interesting model of an economy based on trade and investments of agents. In the mean-field approximation, the model produces a stationary wealth distribution with a power law tail. In this paper we examine characteristic timescales of the model and show that for any finite number of agents, the validity of the mean-field result is time-limited and the model in fact has no stationary wealth distribution. Further analysis suggests that for heterogeneous agents, the limitations are even stronger. We conclude with general implications of the results presented.
\end{abstract}

Keywords: fluctuations (theory), stochastic processes (theory), interacting agent models

ArXiv ePrint: 0809.4139 


\section{Contents}

1. Introduction 2

2. The model and its mean-field solution 2

3. The complete exchange network for a finite $N \quad 3$

4. General exchange network $\quad 7$

4.1. Influence of the initial conditions . . . . . . . . . . . . . . . . . . 9

5. Conclusion 9

$\begin{array}{ll}\text { Acknowledgments } & 10\end{array}$

$\begin{array}{ll}\text { References } & 10\end{array}$

\section{Introduction}

Many empirical studies report broad distributions of income and wealth of individuals and these distributions are often claimed to have power law tails with exponents around 2 for most countries [1]-[5]. The first models attempting to explain the observed properties appeared over fifty years ago [6]-[8]. Much more recently, physicsmotivated kinetic models based on random pairwise exchanges of wealth by agents have attracted considerable interest [9]-[13]. An alternative point of view is adopted in the wealth redistribution model (WRM) where agents continuously exchange wealth in the presence of noise [14]-[16]. There are also several specific effects which can lead to broad wealth distributions [17]-[19]. (For reviews of power laws in wealth and income distributions see [20]-[22], while for general reviews of power laws in science see $[23,24]$.)

In this paper we analyse the WRM with two complementary goals in mind. Firstly we investigate the simplest case when exchanges of all agents are identical, focusing on the validity of the mean-field approximation which is the standard tool for solving the model and deriving the stationary wealth distribution. In particular, we show that for any finite number of agents there is no such stationary distribution (other finite-size effects are discussed for a similar model in [18]). Secondly we investigate the model's behaviour when the network of agent exchanges is heterogeneous. Previous attempts to investigate the influence of network topology on the model [14], [25]-[27] were all based on the mean-field approximation. We show that this is questionable because heterogeneity of the exchange network strongly limits the validity of results obtained using the mean-field approximation.

\section{The model and its mean-field solution}

Adopting the notation used in [14], we study a simple model of an economy which is composed of $N$ agents with wealth $v_{i}(i=1, \ldots, N)$. The agents are allowed to mutually exchange their wealth (representing trade) and they are also subject to multiplicative noise 
(representing speculative investments). The time evolution of agents' wealth is given by the system of stochastic differential equations (SDEs)

$$
\mathrm{d} v_{i}(t)=\left(\sum_{j \neq i} J_{i j} v_{j}(t)-\sum_{j \neq i} J_{j i} v_{i}(t)\right) \mathrm{d} t+\sqrt{2} \sigma v_{i}(t) \mathrm{d} W_{i}(t),
$$

where $\sigma \geq 0$ controls the noise strength. The coefficient $J_{i j}$ quantifies the proportion of the current wealth $v_{j}(t)$ that agent $j$ spends on the production of agent $i$ per unit time. We assume the Itô convention for SDEs and $\mathrm{d} W_{i}(t)$ is standard white noise $[29,30]$. Hence, denoting averages over realizations by $\langle\cdot\rangle$, we have $\left\langle\mathrm{d} W_{i}(t)\right\rangle=0,\left\langle\mathrm{~d} W_{i}(t) \mathrm{d} W_{j}(t)\right\rangle=\delta_{i j} \mathrm{~d} t$, and $\left\langle v_{i}(t) \mathrm{d} W_{i}(t)\right\rangle=0$. By summing $\mathrm{d} v_{i}(t)$ over all agents one can see that the average wealth $v_{\mathrm{A}}(t):=(1 / N) \sum_{i=1}^{N} v_{i}(t)$ is not influenced by wealth exchanges and obeys the SDE $\mathrm{d} v_{\mathrm{A}}(t)=(\sqrt{2} \sigma / N) \sum_{i=1}^{N} v_{i}(t) \mathrm{d} W_{i}(t)$. Therefore $\left\langle\mathrm{d} v_{\mathrm{A}}(t)\right\rangle=0$ and $\left\langle v_{\mathrm{A}}(t)\right\rangle$ is constant. For simplicity we assume $v_{i}(0)=1(i=1, \ldots, N)$ and thus $\left\langle v_{i}(t)\right\rangle=1$ and $\left\langle v_{\mathrm{A}}(t)\right\rangle=1$. (The influence of the initial conditions is discussed in section 4.1.)

The system behaviour is strongly influenced by the exchange coefficients $J_{i j}$. The simplest choice is $J_{i j}=J /(N-1)$ where all exchanges are equally intensive - we say that the exchange network is homogeneous. By rescaling the time we can set $J=1$ which means that during unit time agents exchange all their wealth. Consequently, (1) simplifies to

$$
\mathrm{d} v_{i}(t)=\left(\tilde{v}_{i}(t)-v_{i}(t)\right) \mathrm{d} t+\sqrt{2} \sigma v_{i}(t) \mathrm{d} W_{i}(t)
$$

where $\tilde{v}_{i}(t):=(1 /(N-1)) \sum_{j \neq i} v_{j}$ is the average wealth of all agents but agent $i$. In the limit $N \rightarrow \infty$, fluctuations of $\tilde{v}_{i}(t)$ are negligible and one can replace $\tilde{v}_{i}(t) \rightarrow\left\langle\tilde{v}_{i}(t)\right\rangle=1$ as in [14]. Agents then effectively interact only with the 'mean field' and their wealth levels are independent. Using the Fokker-Planck equation for the wealth distribution $f\left(v_{i}, t\right)$, the stationary solution $f\left(v_{i}\right)$ can be found in the form

$$
f\left(v_{i}\right)=\frac{(\lambda-1)^{\lambda}}{\Gamma(\lambda)} \exp \left[-\frac{\lambda-1}{v_{i}}\right] v_{i}^{-1-\lambda}, \quad \lambda:=1+1 / \sigma^{2} .
$$

For $v_{i} \gg \lambda-1, f\left(v_{i}\right)$ decays approximately as a power law with exponent $2+1 / \sigma^{2}$, while the cumulative distribution has exponent $1+1 / \sigma^{2}$. When $v_{i}$ is well described by (3), we say that the system is in the power law regime.

The empirical studies mentioned above report power law exponents around 2, indicating that in this model, $\sigma \simeq 1$ is needed to obtain realistic power law behaviour of the wealth distribution. In our analytical calculations we assume $\sigma<1$; strong noise $(\sigma \geq 1)$ is discussed separately at the end of the following section.

\section{The complete exchange network for a finite $N$}

To examine when the power law regime is realized, we first investigate the time needed to reach the mean-field solution (3). Such relaxation times were studied very recently using kinetic models of wealth distribution [28].

Given the homogeneous initial conditions $v_{i}(0)=1(i=1, \ldots, N)$, the exchange terms proportional to $\tilde{v}_{i}-v_{i}$ are zero at $t=0$ and can be neglected for small times. Hence when $t$ is small, each $v_{i}(t)$ evolves independently due to multiplicative noise, $v_{i}(t)$ 
is log-normally distributed, and its variance is $\operatorname{var}\left[v_{i}\right](t)=\exp \left[2 \sigma^{2} t\right]-1=2 \sigma^{2} t+\mathrm{O}\left(t^{2}\right)$ : we say that the system is in the free regime. From the known variance $\sigma^{2} /\left(1-\sigma^{2}\right)$ of the mean-field solution (3), we can estimate the time of transition, $t_{1}$, between the free regime and the power law regime as

$$
t_{1}=\frac{1}{2\left(1-\sigma^{2}\right)}
$$

When $t \gg t_{1}$, the system has been given enough time to reach the power law regime.

We now recall the average wealth $v_{\mathrm{A}}(t)$. While $\left\langle\mathrm{d} v_{\mathrm{A}}(t)\right\rangle=0$, one can see that $\left\langle\mathrm{d} v_{\mathrm{A}}^{2}(t)\right\rangle$ is always positive. Hence the variance $\operatorname{var}\left[v_{\mathrm{A}}(t)\right]$ grows without limit, in contrast with the variance of $(3)$ which is finite for $\sigma<1$. To resolve this disagreement we have to accept that $f\left(v_{i}\right)$ as given by (3) is not a stationary solution. But what comes after the power law regime? Since the Fokker-Planck equation for the joint probability distribution $f\left(v_{1}, \ldots, v_{N}\right)$ cannot be solved analytically, we answer this question by investigating the average quantities $\left\langle v_{i}^{2}(t)\right\rangle$ and $\left\langle v_{i}(t) v_{j}(t)\right\rangle(i \neq j)$; now we are considering $\sigma<1$ and hence both are well defined. Due to the assumed homogeneous network of interactions and the chosen initial conditions, all averages $\left\langle v_{i}^{2}(t)\right\rangle$ are identical and the same holds for the cross-terms $\left\langle v_{i}(t) v_{j}(t)\right\rangle$; effectively we are left with only two variables. From the Itô lemma it follows that $\mathrm{d}\left(v_{i}^{2}\right)=\left(2 v_{i}+\mathrm{d} v_{i}\right) \mathrm{d} v_{i}$ and $\mathrm{d}\left(v_{i} v_{j}\right)=v_{i} \mathrm{~d} v_{j}+v_{j} \mathrm{~d} v_{i}+\mathrm{d} v_{i} \mathrm{~d} v_{j}$. After substitution of (2) and averaging over all possible realizations, we obtain the exact set of equations

$$
\begin{aligned}
& \frac{\mathrm{d}\left\langle v_{i}^{2}(t)\right\rangle}{\mathrm{d} t}=2\left[\left\langle v_{i}(t) v_{j}(t)\right\rangle-\left(1-\sigma^{2}\right)\left\langle v_{i}^{2}(t)\right\rangle\right], \\
& \frac{\mathrm{d}\left\langle v_{i}(t) v_{j}(t)\right\rangle}{\mathrm{d} t}=\frac{2}{N-1}\left[\left\langle v_{i}^{2}(t)\right\rangle-\left\langle v_{i}(t) v_{j}(t)\right\rangle\right] .
\end{aligned}
$$

Since we set $v_{i}(0)=1(i=1, \ldots, N),\left\langle v_{i}(t)\right\rangle=1$ and the initial conditions are $\left\langle v_{i}^{2}(0)\right\rangle=1$ and $\left\langle v_{i}(0) v_{j}(0)\right\rangle=1$; for the general case see section 4.1. Independently of the initial conditions, for $\sigma>0$, (5) has only the trivial stationary solution $\left\langle v_{i}^{2}\right\rangle=\left\langle v_{i} v_{j}\right\rangle=0$. This confirms that for a finite $N$, there is no stationary distribution $f\left(v_{i}\right)$.

By solving (5) one obtains the variance $\operatorname{var}\left[v_{i}\right](t)=\left\langle v_{i}^{2}(t)\right\rangle-\left\langle v_{i}(t)\right\rangle^{2}$ as a function of time and as a by-product also the correlation between agents $i$ and $j$

$$
C_{i j}(t):=\frac{\left\langle v_{i}(t) v_{j}(t)\right\rangle-\left\langle v_{i}(t)\right\rangle\left\langle v_{j}(t)\right\rangle}{\sqrt{\operatorname{var}\left[v_{i}(t)\right] \operatorname{var}\left[v_{j}(t)\right]}} .
$$

Since the resulting expressions are rather complicated, here we discuss only their limiting cases. Small time expansions can be found in the form

$$
\begin{aligned}
& \operatorname{var}\left[v_{i}\right](t)=2 \sigma^{2} t+\mathrm{O}\left(t^{2}\right), \\
& C_{i j}(t)=\frac{1}{N-1} t+\mathrm{O}\left(t^{2}\right) .
\end{aligned}
$$

As can be seen, (7) agrees with our previous reasoning about the log-normal nature of $f\left(v_{i}\right)$ in the free regime, while (8) confirms that in the limit $N \rightarrow \infty$, wealth correlations vanish. 
In the limit of large time we obtain

$$
\lim _{t \rightarrow \infty} C_{i j}(t)=1-\sigma^{2}+\frac{\sigma^{2}}{1-\sigma^{2}} \frac{1}{N}+\mathrm{O}\left(1 / N^{2}\right) .
$$

Thus, as $t$ increases, the system passes to the synchronized regime where the wealth of agents is strongly correlated. One can estimate the transition time by comparing the initial linear growth of $C_{i j}$ with its stationary value, leading to

$$
t_{2}=\left(1-\sigma^{2}\right) N+\mathrm{O}(1) .
$$

An alternative estimate can be obtained from $\operatorname{var}\left[v_{i}\right](t)$. Apart from a constant, it contains only terms proportional to $\exp \left[\lambda_{1,2} t\right]$ where

$$
\lambda_{1,2}=\frac{-\sigma^{2}-N\left(1-\sigma^{2}\right) \pm \sqrt{N^{2}\left(1-\sigma^{2}\right)^{2}+2 N \sigma^{2}\left(3-\sigma^{2}\right)-\sigma^{2}\left(4-\sigma^{2}\right)}}{2(N-1)} .
$$

Since $\lambda_{1}<0, \lambda_{2}>0$, and for $\sigma \lesssim 1,\left|\lambda_{1}\right| \gg\left|\lambda_{2}\right|$, the terms proportional to $\exp \left[\lambda_{1} t\right]$ cause the initial saturation of $\operatorname{var}\left[v_{i}\right](t)$ but the terms proportional to $\exp \left[\lambda_{2} t\right]$ eventually take over and cause the divergence of $\operatorname{var}\left[v_{i}\right](t)$. The corresponding transition time can be roughly estimated by solving $\lambda_{2} t=1$, yielding

$$
t_{3}=\frac{1-\sigma^{2}}{2 \sigma^{2}} N+\mathrm{O}(1)
$$

Both $t_{2}$ and $t_{3}$ describe the transition between the power law and synchronized regimes: the former focuses on the growth of correlations, the latter on the growth of variances.

To verify the analytical results presented we investigated the model numerically. For numerical solutions of stochastic differential equations we used Milstein's method [29,31]; random numbers were generated using the standard GSL library and the Mersenne twister generator [32], and the time increment was $10^{-4}$ in all simulations. In the discretization scheme used, there is a non-zero probability that the wealth $v_{i}(t)$ becomes negative [33]. However, thanks to the typical value of $v_{i}(t)$ and the small time step, in the numerical simulations presented this was not an issue. As can be seen in figure 1(a), our analytical results agree with numerical simulations of the system. Due to the small number of agents, transition times $t_{2,3}$ are small and the system goes directly from the free regime to the synchronized regime. In figure 1(b) the number of agents is large and the system behaviour is more complex. In the initial period the variance is small and correlations are negligible, while in the period $t \in[2 ; 300]$ the variance is almost constant and correlations are still small - the system is in the power law regime (due to large computational complexity, no numerical results are shown here). Eventually, for $t \gtrsim 10^{4}$, the synchronized regime is established. The transition times given by (4), (10), and (11) are shown as vertical dotted lines and agree well with the described changes of the system behaviour.

We should sound here a note of caution about the interpretation of the averages $\left\langle v_{i}^{2}(t)\right\rangle$ and $\left\langle v_{i}(t) v_{j}(t)\right\rangle$ and the wealth distribution $f\left(v_{i}, t\right)$. All these quantities are ensemblebased: if many copies of the system evolve independently for time $t$, by examining the final wealth of agent $i$ one can estimate both the distribution $f\left(v_{i}, t\right)$ and the averages. By contrast, when one speaks about an empirical wealth distribution, that is based on the wealth of all agents in one realization only, it is population-based. However, when the number of realizations and the number of agents are large and the wealth correlations 

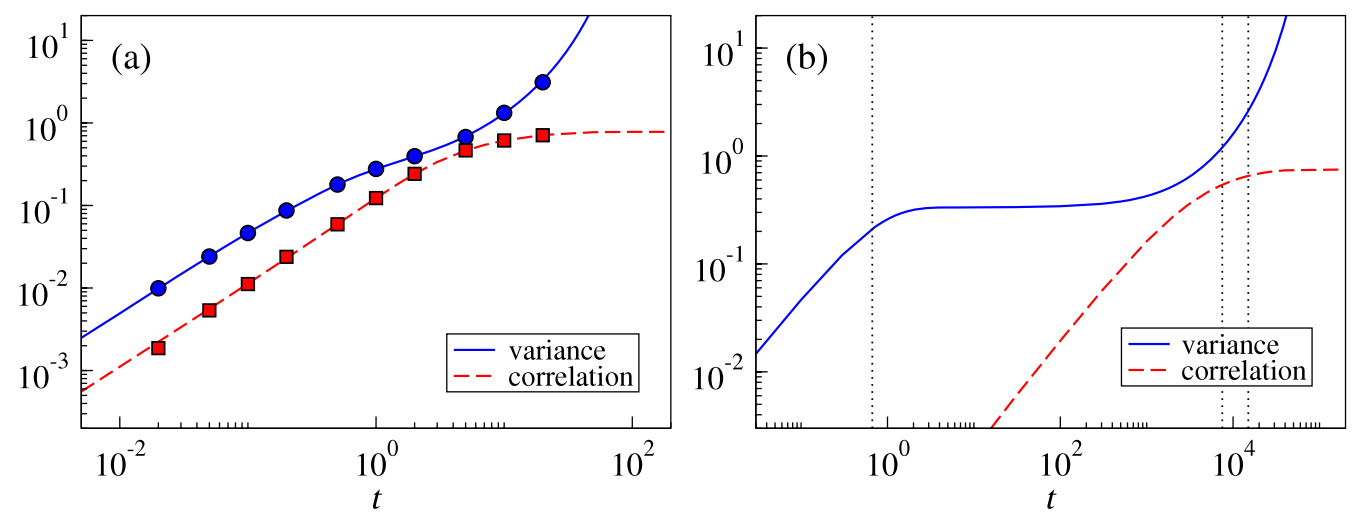

Figure 1. Time evolution of $\operatorname{var}\left[v_{i}\right](t)$ and $C_{i j}(t)$ for 10 agents (a) and for $10^{4}$ agents (b). Analytical results following from (5) are shown as lines; numerical results obtained by averaging over $10^{5}$ realizations are shown as symbols; $\sigma^{2}=$ 0.5. Vertical dotted lines indicate the transition times $t_{1}, t_{2}$, and $t_{3}$, left to right, respectively.

are small, ensemble-based and population-based quantities are alike. Such behaviour was observable also in the numerical simulations presented above. In the free and power law regimes, the variance of wealth in each realization was similar to $\operatorname{var}\left[v_{i}(t)\right]$ (at various times, differences were less than $20 \%$ for $N=10$ and less than $1 \%$ for $N=10000$ ) and its relative fluctuations between realizations were approximately $50 \%$ for $N=10$ and $2 \%$ for $N=10000$. As time goes on, fluctuations of the population-based variance grow and so does the difference between the ensemble-based and population-based variances of wealth. In the synchronized regime, the equivalence of the two quantities breaks entirely.

The nature of the synchronized regime can be better understood by recalling the average wealth $v_{\mathrm{A}}(t)$ again. As explained above, its evolution is given by a sum of multiplicative processes, $\mathrm{d} v_{\mathrm{A}}(t)=(\sqrt{2} \sigma / N) \sum_{i=1}^{N} v_{i}(t) \mathrm{d} W_{i}(t)$. Despite this summation of contributions and their variable strengths $\left(\left\langle v_{i}^{2}(t)\right\rangle\right.$ increases with time), according to figure 2 the distribution of $v_{\mathrm{A}}$ is approximately log-normal and, in agreement with our expectations, the variance $\operatorname{var}\left[v_{\mathrm{A}}\right]$ is increasing. In the initial regime, this increase is due to growing variances of all agents' wealth. In the power law regime, variances of wealth levels are approximately constant but their growing correlations lead to increasing $\operatorname{var}\left[v_{\mathrm{A}}\right]$. In the synchronized regime, wealth correlations are already saturated and the growth of $\operatorname{var}\left[v_{\mathrm{A}}\right]$ is caused by exponentially growing variances of wealths. Since correlations are large, ensemble-based and population-based quantities are no longer equivalent. Finally we remark that since $v_{\mathrm{A}}>0,\left\langle v_{\mathrm{A}}(t)\right\rangle=1$ is fixed, and $\operatorname{var}\left[v_{\mathrm{A}}(t)\right]$ grows without bound, in the course of time it is increasingly probable that $v_{\mathrm{A}}(t)$ will be much smaller than its expected value $\left\langle v_{\mathrm{A}}(t)\right\rangle=1$; this can be interpreted as a high occurrence of temporal depressions of the economy.

When $\sigma \geq 1$, both $\operatorname{var}\left[v_{i}\right](t)$ and $C_{i j}(t)$ diverge and must be replaced by different quantities. Instead of the variance, one can use the mean absolute deviation $\left\langle\left|v_{i}(t)-1\right|\right\rangle^{2}$ which avoids second moments of the wealth distribution and hence can be used for any $\sigma$. Pearson's correlation coefficient can be replaced by a rank correlation coefficient (Kendall's $\tau$ or Spearman's $\rho$ ). All three proposed quantities are hard to handle in analytical 


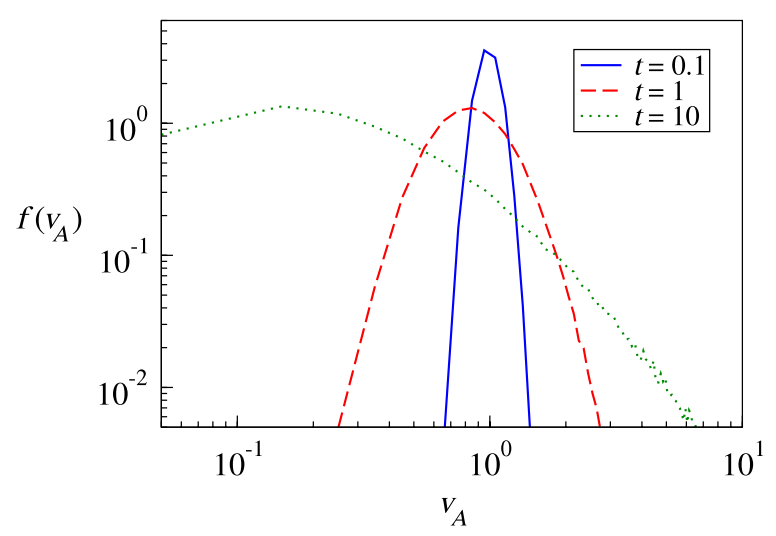

Figure 2. Probability density of the average wealth $v_{\mathrm{A}}$ at various times. Parameter values are $N=10$ and $\sigma^{2}=0.5$; probabilities were obtained from $10^{5}$ independent realizations of the model.

calculations, and with strong noise, numerical simulations of the system are extremely time-demanding. While we have obtained no definite results yet, preliminary outcomes suggest that in this case too the transition from the power law regime occurs at a time proportional to the number of agents $N$.

\section{General exchange network}

Now we generalize the exchange network to an arbitrary graph: denoting the set of neighbours of agent $i$ by $\mathcal{N}_{i}$, the number of neighbours by $k_{i}$, the average number of neighbours by $z$. We assume that each agent interacts equally with all neighbours and per unit time exchanges the whole wealth, and hence

$$
J_{i j}=1 / k_{j} \quad \text { for } i \in \mathcal{N}_{j}, \quad J_{i j}=0 \quad \text { for } i \notin \mathcal{N}_{j} \text {; }
$$

notice that the matrix of exchanges $\mathrm{J}$ is asymmetric. Now, (1) generalizes to

$$
\mathrm{d} v_{i}=\left(\hat{v}_{i}-v_{i}\right) \mathrm{d} t+\sqrt{2} \sigma v_{i} \mathrm{~d} W_{i}
$$

where $\hat{v}_{i}:=\sum_{j \in \mathcal{N}_{i}} v_{j} / k_{j}$. By averaging over realizations we obtain the set of equations for the stationary values of the average wealths

$$
\left\langle v_{i}\right\rangle=\sum_{m \in \mathcal{N}_{i}} \frac{\left\langle v_{m}\right\rangle}{k_{m}}
$$

which is solved by $\left\langle v_{i}\right\rangle \sim k_{i}$. Assuming average wealth equal to 1 , (14) has the unique solution $\left\langle v_{i}\right\rangle=k_{i} / z$. This means that the topology of the exchange network is crucial for the distribution of wealth among the agents. Consequently, when $\sigma$ is small and hence wealth fluctuations are negligible, a power law distribution of wealth can be purely a topological effect of a scale-free degree distribution in the network of agent exchanges. 
To proceed, $\left\langle v_{i}^{2}(t)\right\rangle$ and $\left\langle v_{i}(t) v_{j}(t)\right\rangle$ are again the key quantities. They fulfil the equations

$$
\begin{aligned}
& \frac{\mathrm{d}\left\langle v_{i}^{2}\right\rangle}{\mathrm{d} t}=2 \sum_{m \in \mathcal{N}_{i}} \frac{\left\langle v_{i} v_{m}\right\rangle}{k_{m}}-2\left(1-\sigma^{2}\right)\left\langle v_{i}^{2}\right\rangle, \\
& \frac{\mathrm{d}\left\langle v_{i} v_{j}\right\rangle}{\mathrm{d} t}=\sum_{m \in \mathcal{N}_{i}} \frac{\left\langle v_{j} v_{m}\right\rangle}{k_{m}}+\sum_{n \in \mathcal{N}_{j}} \frac{\left\langle v_{i} v_{n}\right\rangle}{k_{n}}-2\left\langle v_{i} v_{j}\right\rangle,
\end{aligned}
$$

which can be derived similarly to (5). We set the initial conditions according to the stationary wealths as $v_{i}(0)=k_{i} / z$ and thus $\left\langle v_{i}^{2}(0)\right\rangle=k_{i}^{2} / z^{2}$ and $\left\langle v_{i}(0) v_{j}(0)\right\rangle=k_{i} k_{j} / z^{2}$ (the general case is studied in section 4.1). From (15) it follows that

$$
\left.\frac{\mathrm{d}\left\langle v_{i}^{2}\right\rangle}{\mathrm{d} t}\right|_{t=0}>0,\left.\quad \frac{\mathrm{d}\left\langle v_{i} v_{j}\right\rangle}{\mathrm{d} t}\right|_{t=0}=0,
$$

which means that the growth of $\operatorname{var}\left[v_{i}\right](t)$ precedes the growth of $C_{i j}(t)$. This gives us a way to investigate the small time behaviour of (15): assuming $\left\langle v_{i}(t) v_{j}(t)\right\rangle$ constant, we obtain $\left\langle v_{i}^{2}(t)\right\rangle$ which in turn leads to an enhanced estimate of $\left\langle v_{i}(t) v_{j}(t)\right\rangle$. For neighbouring agents $i$ and $j$, the results are

$$
\begin{aligned}
& \operatorname{var}\left[v_{i}(t)\right]=\frac{2 \sigma^{2} k_{i}^{2}}{z^{2}} t+\mathrm{O}\left(t^{2}\right), \\
& C_{i j}(t)=\frac{k_{i}+k_{j}}{2 k_{i} k_{j}} t+\mathrm{O}\left(t^{2}\right) .
\end{aligned}
$$

Moreover, it can be shown that when the shortest path between agents $i$ and $j$ has the length $L$, the leading term of $C_{i j}(t)$ is proportional to $t^{L}$. These results are confirmed by figure 3 where we investigate a system of ten agents who are placed on a ring (i.e., $k_{i}=2$, $i=1, \ldots, 10)$. As can be seen, numerical results agree well with $C_{i j}(t)$ proportional to $t^{L}$. The system produces a 'cascade' of correlations: first only neighbouring agents are considerably correlated, then also agents with the distance 2, distance 3, and so forth.

For (13), the mean-field approximation yields the stationary distribution

$$
f_{i}\left(v_{i}\right)=K_{i} \exp \left[-\left(\lambda_{i}^{\prime}-1\right) / v_{i}\right] v_{i}^{-2-\lambda_{i}^{\prime}}
$$

where $\lambda_{i}^{\prime}=1+k_{i} /\left(z \sigma^{2}\right)$ and the corresponding variance is $\operatorname{var}\left[v_{i}\right]=k_{i}^{2} \sigma^{2} /\left(z^{2}\left(1-\sigma^{2}\right)\right)$. By comparing this stationary variance with (17), we obtain the time of transition from the free regime to the power law regime as

$$
t_{1}^{\prime}=\frac{1}{2\left(1-\sigma^{2}\right)}
$$

which is identical to (4). Further, from (18) we see that the time of transition from the power law regime to the synchronized regime is proportional to $k_{i} k_{j} /\left(k_{i}+k_{j}\right)$ and thus for the whole network it can be estimated as

$$
t_{2}^{\prime}=\mathrm{O}(z)
$$

which is a generalization of (10). We see that for networks with a relatively small average degree, the power law regime appears only for a limited time or not at all. 


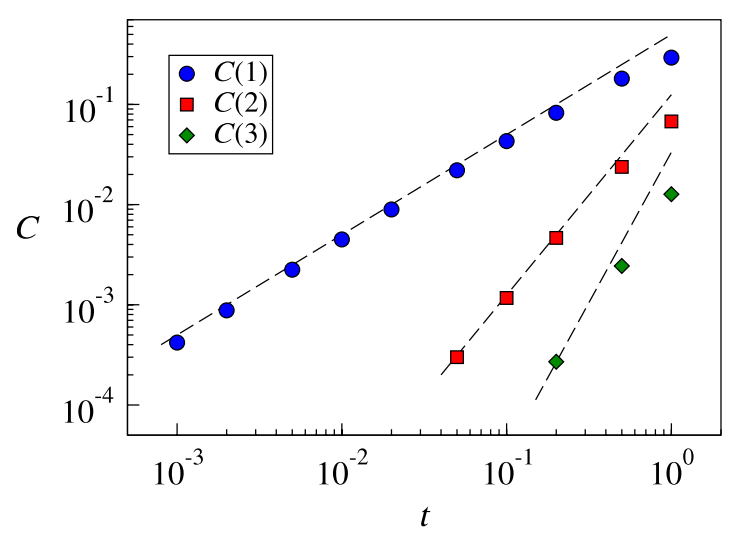

Figure 3. Time evolution of correlations for the ring network of 10 agents, $\sigma^{2}=0.25$. Symbols show numerical results for neighbouring agents (circles), agents with the distance 2 (squares), and agents with the distance 3 (diamonds), averaged over $10^{7}$ realizations. Dashed lines have slopes 1,2 , and 3 , respectively.

We were unable to obtain an equivalent of the transition time $t_{3}$ for a general network. Considering, for example, a simple star-like structure with one agent in the centre and the remaining $N-1$ agents connected only to that agent, one can see that the transition time $t_{3}^{\prime}$ is small and does not scale with $N$. This suggests that, like $t_{2}^{\prime}, t_{3}^{\prime}$, it is $\mathrm{O}(z)$. This contradicts the findings presented in [14] (page 541) where they report stationary power law tails for $z=4$; it is possible that their numerical results are influenced by finite-time and finite-size effects.

\subsection{Influence of the initial conditions}

There is still one more transition time to investigate. When the initial conditions $v_{i}(0)$ are not set in line with the stationary wealths given by equation (14), a certain time is needed to redistribute the excessive wealth levels over the network; we say that the system is in the equilibration regime. Since $\left\langle\mathrm{d} W_{i}\right\rangle=0$, noise terms do not contribute to the redistribution. Thus, (13) effectively simplifies to $\mathrm{d} v_{i}=\left(\hat{v}_{i}-v_{i}\right) \mathrm{d} t$ which leads to the exponential convergence of $v_{i}$ to the stationary value $k_{i} / z$. By making the substitution $u_{i}:=v_{i}-k_{i} / z$ we obtain

$$
\dot{u}_{i}+u_{i}-\sum_{j \in \mathcal{N}_{i}} \frac{u_{j}}{k_{j}}=0
$$

whose timescale is given by the initial terms $\dot{u}_{i}+u_{i}$ as $\mathrm{O}(1)$. Thus, the initial wealth distribution equilibrates in time $\mathrm{O}(1)$. Since the transition from the free regime occurs roughly at the same time, the system passes from the equilibration regime directly to the power law regime.

\section{Conclusion}

We have shown that for the model investigated agent wealths have no stationary distribution and the power law tailed distribution reported in previous works is only 
transient. In addition, for any finite number of agents, their average wealth $v_{\mathrm{A}}$ follows a multiplicative process with a fixed expected value $\left\langle v_{\mathrm{A}}\right\rangle$ and an increasing variance $\operatorname{var}\left[v_{\mathrm{A}}\right]$. Hence, as illustrated in figure 2, the probability $P\left(v_{\mathrm{A}}<x\right)$ approaches 1 for any $x>0$. We can conclude that the simple economy produced by the model is an uneasy one: the longer it evolves, the higher the probability that a given agent $i$ has wealth much smaller than any positive fraction of the expected wealth $\left\langle v_{i}\right\rangle$.

There is also a more general lesson to be learnt. In essence, the mean-field approximation here anchors the agent wealths to their expected values and thus weakens the diffusive nature of the stochastic system studied. Mathematically speaking, the system behaviour depends on the order of limits $N \rightarrow \infty$ and $t \rightarrow \infty$ : in the former case there is a stationary wealth distribution; in the latter case there is none. This is an undesired consequence of the mean-field approximation which, as with other stochastic models, should be used with great caution. In particular, when using it, one should check whether the nature of the system studied is not changed. To achieve this, in this paper we have used an aggregate quantity (the average wealth) and a quantity obtained using the mean-field approximation (the wealth variance).

On the other hand, in some cases an anchoring term may be appropriate. For example, a simple taxation of wealth can be achieved by introducing the term $r\left(1-v_{i}\right) \mathrm{d} t$ to $(2)$, where $r>0$ represents the tax rate. Then the set of equations for $\left\langle v_{i}^{2}(t)\right\rangle$ and $\left\langle v_{i}(t) v_{j}(t)\right\rangle$ has a non-trivial stationary solution for $\sigma<1$; one can say that the proposed taxation stabilizes the system. Notably, systems of coupled stochastic equations with multiplicative noise and negative feedback are common in the study of non-equilibrium phase transitions in magnetic systems [34]. Our work shows that this negative is crucial for mean-field studies of such systems [35].

In addition to the results presented, several questions remain open. First, for large time $t$, the analytical form of the wealth distribution $f\left(v_{i}, t\right)$ is unknown. Second, for an arbitrary network of exchanges, the limiting value of the correlation $C_{i j}(t)$ and also the transition time $t_{3}^{\prime}$ are of interest. Third, the strong noise case deserves more attention and perhaps an attempt for approximate analytical results. Finally, the model studied is simplistic, since it combines two ingredients of an economy - trade and speculation - in a very unrealistic way. Devising a more adequate model remains a future challenge.

\section{Acknowledgments}

We acknowledge the hospitality of the Comenius University (Bratislava, Slovakia) and the Fribourg University (Fribourg, Switzerland). We cordially thank František Slanina for early discussions and Zoltán Kuscsik and Joseph Wakeling for helpful comments.

\section{References}

[1] Pareto V, 1897 Cours d'economie politique (Rouge: Lausanne)

[2] Piggott J, 1984 Econ. Record 60252

[3] Aoyama H et al, 2000 Fractals 8293

[4] Drăgulescu A and Yakovenko V M, 2001 Physica A 299213

[5] Sinha S, 2006 Physica A 359555

[6] Champernowne D G, 1953 Econ. J. 63318

[7] Wold H O A and Whittle P, 1957 Econometrica 25591

[8] Stiglitz J E, 1969 Econometrica 37382

[9] Ispolatov S et al, 1998 Eur. Phys. J. B 2267 
[10] Drăgulescu A and Yakovenko V M, 2000 Eur. Phys. J. B 17723

[11] Slanina F, 2004 Phys. Rev. E 69046102

[12] Patriarca M et al, The ABCD's of statistical many-agent economy models, 2006 arXiv:physics/0611245

[13] Chatterjee A and Chakrabarti B K, 2007 Eur. Phys. J. B 60135

[14] Bouchaud J-P and Mézard M, 2000 Physica A 282536

[15] Solomon S and Richmond P, 2001 Physica A 299188

[16] Di Matteo T et al, 2004 The Physics of Complex Systems (New Advances and Perspectives) ed F Mallamace and H E Stanley (Amsterdam: IOS Press)

[17] Sornette D, 1998 Phys. Rev. E 574811

[18] Huang Z-F and Solomon S, 2001 Physica A 294503

[19] Reed W J, 2001 Econ. Lett. 7415

[20] Quadrini V and Ríos-Rull J-V, 1997 Models of the Distribution of Wealth Research Department, Federal Reserve Bank of Minneapolis

[21] Davies J B and Shorrocks A F, The distribution of wealth, 2000 Handbook of Income Distribution (Handbooks in Economics) ed A B Atkinson and F Bourguignon (Amsterdam: North-Holland)

[22] Yakovenko V M, Statistical mechanics approach to econophysics, 2007 Springer Encyclopedia of Complexity and System Science (Berlin: Springer)

[23] Newman M E J, 2005 Contemp. Phys. 46323

[24] Farmer J D and Geanakoplos J, Power laws in economics and elsewhere, 2006 SFI Technical Report

[25] Souma W et al, Small-world effects in wealth distribution, 2001 arXiv:cond-mat/0108482

[26] Garlaschelli D and Loffredo M I, 2004 Physica A 338113

[27] Garlaschelli D and Loffredo M I, 2008 J. Phys. A: Math. Theor. 41224018

[28] Gupta A K, 2008 Physica A 3876819

[29] Gardiner C W, 2004 Handbook of Stochastic Methods 3rd edn (Berlin: Springer)

[30] Van Kampen N G, 2007 Stochastic Processes in Physics and Chemistry 3rd edn (Amsterdam: North-Holland)

[31] Higham D J, 2001 Siam Rev. 43525

[32] Galasi M et al, 2002 GNU Scientific Library (Reference Manual) (Bristol: Network Theory Ltd)

[33] Moro E, 2004 Phys. Rev. E 70 045102(R)

[34] Birner T et al, 2002 Phys. Rev. E 65046110

[35] Muñoz MA et al, 2005 Phys. Rev. E 72056102 